\title{
Publisher Correction: Switching with ions
}

Morgan Trassin (10) and John T. Heron (D)

Correction to: Nature Nanotechnology https://doi.org/10.1038/s41565-021-00938-9, published online 29 July 2021.

In the version of this News \& Views article originally published online, the title was incorrectly shown as 'Switching with ions inversion'; it has now been corrected to 'Switching with ions' in all versions of the article.

Published online: 19 August 2021

https://doi.org/10.1038/s41565-021-00978-1

(C) Springer Nature Limited 2021 